\title{
ELM pacing and trigger investigations at JET with the new ITER-Like Wall
}

\author{
P. T. Lang 1), D. Frigione 2), A. Géraud 3), T. Alarcon 3), P. Bennett 4), G. Cseh 5), \\ D. Garnier 3), L. Garzotti 4), F. Köchl 6), G. Kocsis 5), M. Lennholm 7), R. Neu 8), \\ R. Mooney 4), S. Saarelma 4), B. Sieglin 1), and JET EFDA Contributors*
}

JET-EFDA, Culham Science Centre, Abingdon, OX14 3DB, UK

1) Max Planck Institute for Plasma Physics, EURATOM Association, Boltzmannstr. 2, 85748

Garching, Germany

2) Associazione EURATOM-ENEA sulla Fusione, CP 65, Frascati, Rome, Italy

3) CEA, IRFM, 13108 Saint Paul-Lez-Durance, France

4) EURATOM/CCFE Fusion Association, Culham Science Centre, Abingdon, Oxon, OX14 3DB, UK

5) WIGNER RCP RMI, EURATOM Association, POB 49, 1525 Budapest, Hungary

6) Association EURATOM-ÖAW/ATI, Atominstitut, TU Wien, 1020 Vienna, Austria

7) JET-EFDA CSU, Culham Science Centre, Abingdon, Oxon OX143DB, UK

8) EFDA-CSU, Boltzmannstr.2, 85748 Garching, Germany

*See the Appendix of F. Romanelli et al., Proceedings of the 24th IAEA Fusion Energy

Conference 2012, San Diego, US

E-mail contact of main author: peter.lang@ipp.mpg.de

\begin{abstract}
During the installation of the new ITER-Like Wall at JET, the High Frequency Pellet Injector has been further improved. The launching system is now capable of delivering reliable fuelling size pellets from the magnetic outboard side up to $15 \mathrm{~Hz}$ repetition rate. Pacing size pellets can be produced at rates up to $50 \mathrm{~Hz}$ but pellet trains suffer some losses during the transfer to the plasma. A significant fraction of the pellet train can arrive at the plasma when launched from the outboard, while only a few pellets make it to the vessel inboard launching site. Stable and reliable ELM control was achieved when using outboard fuelling size pellets. This tool was successfully applied for scenario development purposes in the ITER baseline H-mode scenario at 2.5 MA. Employed for ELM sustainment and impurity control, pellets prevented the ELM frequency from becoming so low as to cause a radiative collapse of the discharge. Despite technical limitations, injecting outboard pacing size pellets resulted in a transient enhancement of the initial ELM frequency up to a factor 4.5. This could be achieved in cases where a continuous train of sufficiently large and fast pellets were arriving in the plasma at a frequency of up to $31 \mathrm{~Hz}$. Pacing size pellets were also used to investigate the ELM trigger threshold. Three basic parameters could be identified for outboard pellet launch. The ELM triggering probability increased with: i) the time elapsed since the previous ELM occurred, ii) pellet mass and iii) pellet speed. An indication for dependence of the ELM trigger threshold on the poloidal pellet launch position has been found; inboard launched pellets seem to reveal a higher trigger capability than pellets launched from the outboard. Finally, we compared the pellet penetration depth required for ELM triggering in the actual JET configuration with plasmafacing components to similar previous experiments performed with a carbon wall. This comparison indicates that pellet ELM triggering requires deeper penetration in the ILW configuration.
\end{abstract}

\section{Introduction}

Controlling the ELM frequency is an important task for the development of high performance plasma scenarios. ELM control requirements in ITER [1] cover the limitation and mitigation of large heat fluxes to the divertor and the first wall expected during type-I ELMs for H-mode operational scenarios at high plasma current. Furthermore, the sustainment of a minimum ELM frequency, even in the low current regime, is required when operating with a $\mathrm{W}$ divertor at low density, to expel tungsten (W) from the plasma edge before it diffuses to the plasma core [2]. Following its first demonstration at ASDEX Upgrade [3,4,5] and the recent successful achievement of an ITER relevant 10x ELM frequency increase on DIII-D [6], pellet ELM pacing is considered as one of the most promising tools for ELM control in ITER. ELM triggering occurs most likely due to the local impact of the pellet ablation plasmoid in the edge barrier, which is expected to be effective even in high performance and high plasma 
current scenarios. Besides allowing ELM-pacing investigations under the most ITER relevant conditions, JET also allows further investigations of ELM triggering conditions and their underlying physics to provide a sound basis for predicting the potential ELM mitigation capability in ITER. To exploit this opportunity, a High Frequency Pellet Injector (HFPI) system is being optimized at JET [7]. The main goal of the HFPI is to demonstrate ELM pacing in a large size tokamak with a tenfold increase in ELM frequency with a minimum of confinement degradation. This injector should furthermore shed light on the required ELM trigger conditions and help to find the smallest possible pellet mass still needed for triggering. Thus, the pellet imposed unwanted fuelling burden causing also additional convective confinement losses can be minimized.

\section{Setup and experimental boundary conditions}

Experiments reported here were performed in 2012 during the first year of operation at JET with the newly installed ITER-Like Wall (ILW). This metallic wall was installed, mainly by remote handling, in a major shutdown 2009-2011. The previous carbon (C) plasma-facing components were replaced by a combination of beryllium $(\mathrm{Be})$ in the main chamber and tungsten (W) in the divertor, as foreseen for ITER. The main goal, to reduce fuel retention rates by an order of magnitude, was achieved [8] while the plasma $\mathrm{C}$ content was reduced by, on average, a factor of 20 . For the new, all-metal environment, some significant changes were observed with respect to the carbon dominated regime [9]. A comparable sharp drop as in the $\mathrm{C}$ edge fluxes is also detectable in $Z_{\text {eff }}$ which reduces in average from 1.9 to 1.2 with change of the main wall material [10]. Besides the reduction in $\mathrm{C}$, oxygen levels were also reduced by roughly one order of magnitude.

To exploit this new wall, a careful optimization of the control of metallic impurity sources and heat loads was required to re-establish high confinement scenarios. The ELM frequency is the main factor for the control of metallic impurity influx and accumulation. The baseline type-I ELMy H-mode regime was re-established avoiding $\mathrm{W}$ accumulation as reported in [11]. In this study it became clear that deuterium had to be puffed at a significant rate (typically above $10^{22} \mathrm{D} / \mathrm{s}$ ) in the divertor during the main heating phase to achieve stable conditions with respect to central radiation peaking. Energy confinement tends to be lower with the new wall, especially in highly shaped plasmas at high gas fuelling [12]. This is mainly attributed to lower edge pedestal temperatures [13]. Type-I ELMs in high deuterium fuelling pulses, at low pedestal temperatures, exhibit a much slower crash of the edge electron temperature than similar C wall cases and, consequently, a slower rise in divertor ELM heat load and reduced surface peak temperatures for a given drop in stored energy. As with the baseline $\mathrm{H}$-modes, the operation range is somewhat restricted by $\mathrm{W}$ accumulation in discharges with little gas injection [14].

Pellet pacing and trigger investigations presented in this paper employed reference baseline scenarios only. A scenario with low triangularity at $\mathrm{I}_{\mathrm{P}}=2.0 \mathrm{MA}$ able to be run steadily at an ELM frequency $\mathrm{f}_{\mathrm{ELM}}$ of about $8 \mathrm{~Hz}$ was chosen for experiments applying small pellets. For pacing and ELM sustainment demonstration in plasmas at $\mathrm{I}_{\mathrm{P}}=2.5 \mathrm{MA}$ at low and high triangularity large pellets had to be used. The obtained results can be compared to recent findings from similar experiments performed with a $\mathrm{C}$ wall [15].

The pellets were produced by the HFPI, which was installed on JET at the end of 2007 and further improved during the shutdown by modifications of the extrusion nozzle assembly eliminating the ice extrusion instability causing a general degradation of performance [7]. The HFPI is designed to launch pellets from three different injection locations for fuelling and ELM pacing purposes with variable size and speed. The pellet size and hence the nominal particle contend is preselected by the diameter (fixed at $4 \mathrm{~mm}$ for fuelling, $1.5 \mathrm{~mm}$ for pacing size) and the variable length, the speed by the applied propellant gas pressure. A sketch of the experimental set up as used during the first campaigns with the ILW is shown in figure 1. A 
single screw extruder produces either one continuous large ribbon of ice for the production of fuelling size pellets or two simultaneous continuous narrow ribbons to provide pacing size pellets. A set of electromagnetic cutters and fast valves is used to respectively cut and accelerate the pellets. The high injection frequency for the small pellets is obtained by cutting pellets alternately from the two small ribbons of ice. The maximum ice production rate of the installed system is $1400 \mathrm{~mm}^{3} / \mathrm{s}$, consistent with the delivery of large pellets at $15 \mathrm{~Hz}$, the most demanding mode in terms of ice flow. The diameter of the small pellets was increased from $1.2 \mathrm{~mm}$ to $1.5 \mathrm{~mm}$ in order to compensate for the strong erosion occurring in the injection line and the unusually long and strongly bended pellet flight tubes. Despite these minor modifications, the injector remains very similar to the prototype designed at the beginning of the project [16].

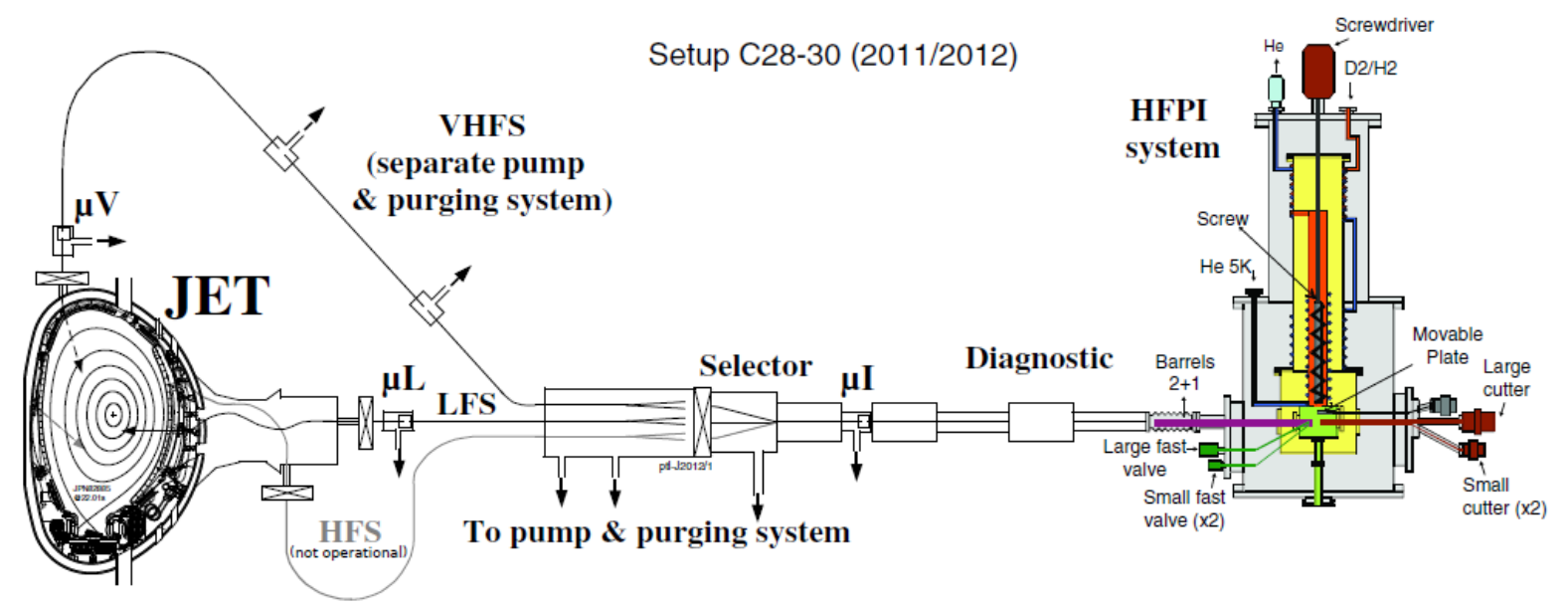

Figure 1: High Frequency Pellet Injector after the modernization performed simultaneously with the installation of the ITER like wall (ILW). Set up during campaigns C28-C30.

The pellet injector is connected to the JET torus through an injection line and different flight tubes. The injection line allows, in its first stage, the pumping of the propellant gas while transferring pellets to the entrance of a fast 4-way selector able to change within less than 50 ms between any of its positions; one pellet dump (not shown in the figure) and three flight tubes. The Low Field Side (LFS) position is located about $9 \mathrm{~m}$ downstream from the injector exit; the last section of this flight tube which is installed inside the new ITER like ICRH antenna, is a bit tortuous due to the geometry of the antenna. About $18 \mathrm{~m}$ transfer is required to reach the Vertical High Field Side (VHFS) injection position. A separate pumping and purging system is attached to this section to pump the gas produced by the pellet erosion inside the flight tubes. Operation of the High Field Side (HFS) injection position is not yet possible. The pellet injection system is equipped with a set of diagnostics to measure the injection parameters. A CCD camera monitors quality and dimensions of the extruded ice as well as the extrusion speed. A set of light barriers measure the pellet velocity at the injector exit. Several microwave cavities are installed for pellet size measurement. An initial pellet size measurement is performed by two cavities, tuned for small and large pellets respectively (location indicated as $\mu \mathrm{I}$ in figure 1). Additional single cavities are installed at the end of both the LFS (indicated as $\mu \mathrm{L}$ ) and the VHFS flight tubes (indicated as $\mu \mathrm{V}$ ). According to their primarily assigned purposes, the fuelling VHFS track microwave cavity is tuned for large pellets while the pacing LFS track cavity is optimized to monitor small pellets. This set of diagnostics has enabled the localization of zones with higher risk of pellets breaking up and the estimation the erosion/slowing down rates during the pellet transit from the injector to the plasma. 
For monitoring the ablating pellet in the plasma the visible light emitted by the pellet cloud particles is recorded by a wide angle view diode and a fast framing camera. The photodiode's viewing cone contains only the plasma regions where the VHFS injected pellets are ablated. The view of the fast framing observation covers only the ablating LFS pellets. Additionally, there is only a small angle between the optical axis of the fast framing camera view and the pellet path (LFS), moreover the spatial resolution is limited by the operational modus of fast framing. Therefore, this observation does not allow for pellet tracking and penetration depth measurements. However, it can be applied as ablation monitor by integrating the frames over the region of interest around the pellet ablation region. This way, pellet radiation versus time with a temporal resolution of the framing rate (typically $10-70 \mathrm{kHz}$ ) - and the pellet lifetime can be obtained.

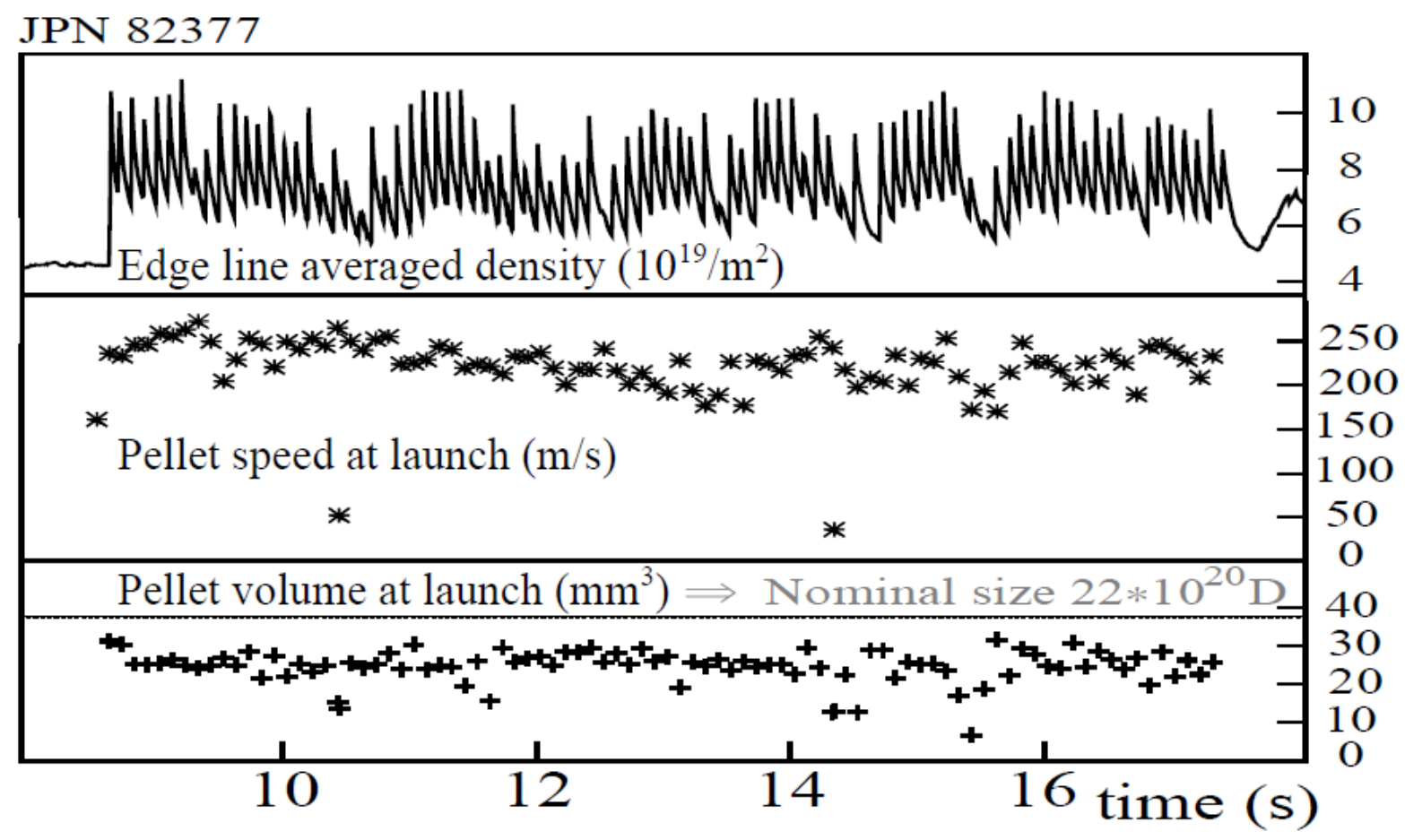

Figure 2: Monitoring discharge showing reliable delivery of fuelling size pellets launched at $10 \mathrm{~Hz}$. Operating in L-mode allows determining the amount of pellet deposited particles.

The pellet launching system can currently be operated either in a configuration using large pellets with a repetition rate up to $15 \mathrm{~Hz}$ or up to $50 \mathrm{~Hz}$ with much smaller pacing size pellets. More than $90 \%$ of the requested pellets are reliably fired when operating with one specific fuelling setting at $15 \mathrm{~Hz}$ (nominal size $22 \times 10^{20} \mathrm{D}$, launch speed $150 \mathrm{~m} / \mathrm{s}$ ). An example is shown in figure 2; displaying results from a discharge (JPN82377) solely run for monitoring the arriving pellets. Stable L-mode conditions with moderate auxiliary heating preventing too strong cooling by the pellets, allow precise analysis of the pellet mass deposited inside the last closed flux surface while avoiding the disturbing influence of ELM activity. For the case shown, a train of pellets was requested at a frequency of $10 \mathrm{~Hz}$ for 9 s. 87 out of these 90 pellets arrived in the plasma, corresponding to $97 \%$. Taking only pellets delivering more than half of the average mass, 79 pellets are counted (88\%).

For small pellets also one specific pacing setting could be established which worked well at $50 \mathrm{~Hz}\left(2.1 \times 10^{20} \mathrm{D}, 170 \mathrm{~m} / \mathrm{s}\right)$. However, the installed transfer system causes a reduced performance inside the torus. While virtually all the launched fuelling pellets arrive in the plasma when they are injected from the outboard (LFS) only $30-50 \%$ of pacing size pellets make it through the same flight line. These pacing pellets furthermore arrive with reduced 
speed $(60-110 \mathrm{~m} / \mathrm{s})$ and showing a significant mass scatter. From monitoring the particle inventory of small pellets in L-mode discharges it was seen that only the largest pellets arriving in the plasma reached the design value. Furthermore, launch speed variations and different deceleration in the tube results in a significant variation in the intervals between successive pellets arriving at the plasma. When using the vertical inboard launch (VHFS) track, only few pacing size pellets arrive in the plasma, ruling out ELM pacing with pacing pellets via this flight tube. Enough pacing pellets do, however, enter the plasma through the VHFS track to allow ELM triggering investigations. For fuelling pellets, on the other hand, significant pellet fragmentation prevented efficient VHFS injection. An overview of the available pellet types and the injection lines with their typical applications in this study is provided in Table I.

\begin{tabular}{|c|l|l|}
\hline \multicolumn{1}{|c|}{ Pellet size } & Small (pacing) & Large (fuelling) \\
\hline Pellet track & $\begin{array}{l}\text { Single pellets for trigger } \\
\text { investigations }\end{array}$ & Not possible \\
\hline VHFS & $\begin{array}{l}\text { ELM pacing up to 30 Hz } \\
\text { (delivery efficiency up to 0.5) }\end{array}$ & $\begin{array}{l}\text { ELM sustainment up to 15 } \\
\text { Hz (delivery efficiency up to 1.0) }\end{array}$ \\
\hline LFS & \multicolumn{2}{|c|}{ Not operational } \\
\hline HFS
\end{tabular}

Table I: Typical applications of available pellet sizes and injection lines.

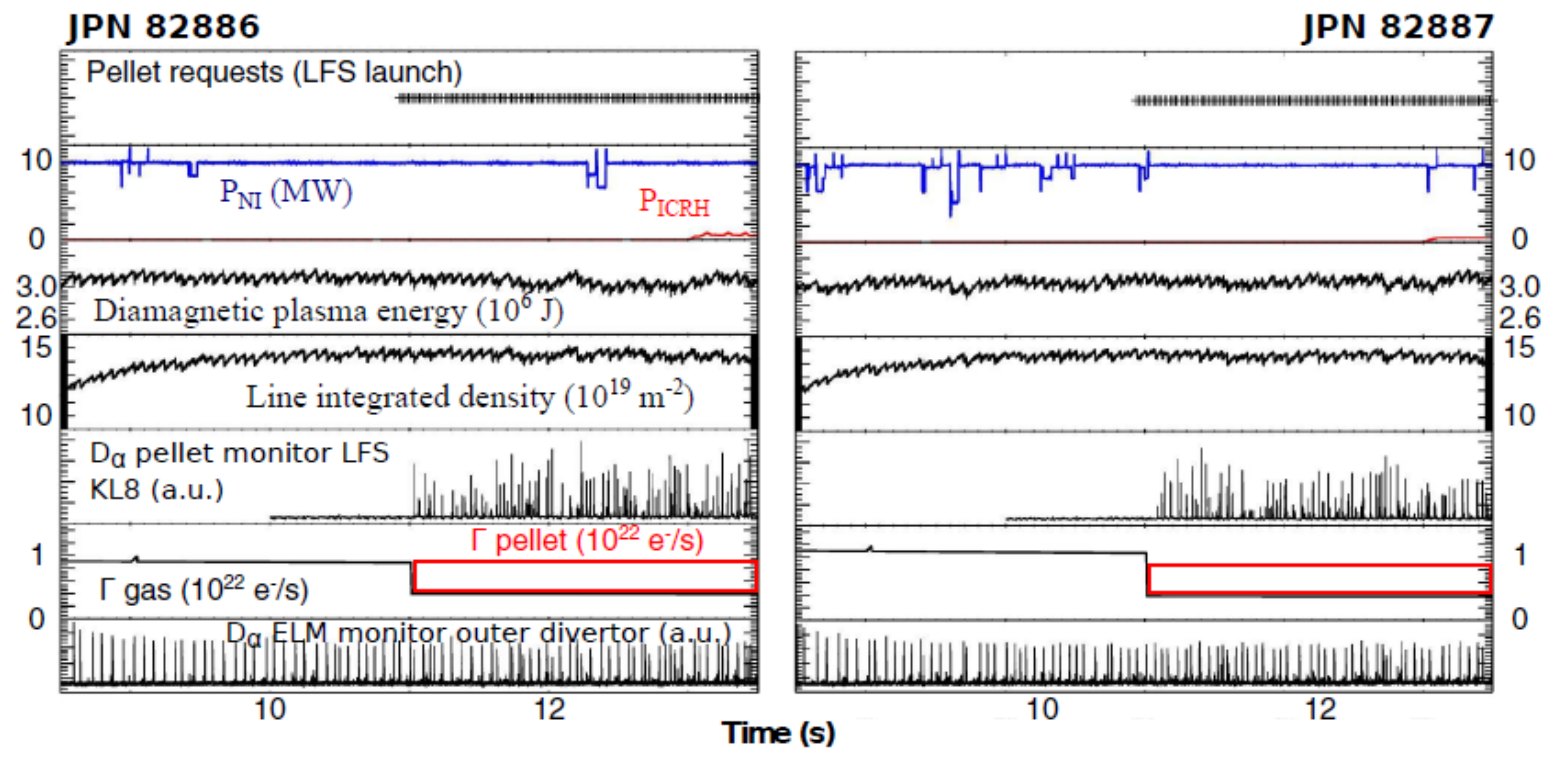

Figure 3: Matching discharges - total particle flux to plasma (left) or ELM frequency (right). Compared to gas puffing less pellet carried particle flux is required to achieve the same impact on the ELM frequency.

\section{ELM pacing investigation}

Dedicated pacing experiments applying small LFS pellets were carried out using the reference baseline scenario with low triangularity as our pacing reference scenario $\left(\mathrm{I}_{\mathrm{P}}=2.0 \mathrm{MA}, \mathrm{B}_{\mathrm{t}}=\right.$ $2.1 \mathrm{~T}, \mathrm{q}_{95}=3.3$; lower and upper triangularities $\delta^{1}=0.36$ and $\delta^{\mathrm{u}}=0.19$, respectively). This configuration was found capable to sustain without impurity accumulation and radiation induced confinement degradation even at low heating power (10 MW NI heating) and gas flux $\left(0.5 \times 10^{22} \mathrm{D} / \mathrm{s}\right)$ at low ELM frequencies of $6-8 \mathrm{~Hz}$. Pellets were seen to cause an increase of the ELM frequency with increasing pellet rate, however no further reduction was 
possible of the remaining minimum gas puff required to avoid impurity accumulation. The ELM frequency is increased above the ELM frequency achieved in a gas fuelled reference experiment, demonstrating that pellet injection induces a stronger ELM frequency enhancement than a similar amount of particles introduced via gas puffing. However, at a comparable ELM frequency the impact on confinement, core density and ELM peak power load is the same with pellet pace making as with gas puffing. This is shown in figure 3 , the left case shows an example where two phases within a discharge are matched with respect to the applied fuelling flux - the first one with pure gas puffing and the second one with a pacing size pellet sequence combined with a small residual gas puff. During the second phase the ELM frequency raises from 13 to $20 \mathrm{~Hz}$. In order to match the ELM frequency (right case in figure 3) the gas flux (second box from bottom) in the first phase had to be increased slightly from 0.9 to $1.1 \times 10^{22} \mathrm{e}^{-} / \mathrm{s}$. From the temporal evolution it can be seen that many ELMs are directly triggered by the pellets, i.e. by the direct interaction while the pellet ablation is still on going. For sequences where several consecutive pellets arrive with consistent shape and speed, direct ELM pacing was found, increasing the ELM frequency by a factor up to 4.5 with respect to the initial value. Apparently, restrictions in the durations of these pacing phases are due to technical limitations on arrival reliability and speed/size of the arriving pellets. Applying pacing pellets to increase the ELM rate had no significant impact on plasma density or confinement.

This is shown for the best case achieved so far in figure 4, displaying (from bottom to top) the time traces of the applied heating power, the diamagnetic plasma energy, the deuterium injection rate (and the particle flux delivered by a perfect $50 \mathrm{~Hz}$ train of pellets with full nominal size, the real pellet carried particle flux is transiently at most $2 / 3$ of this value), the line integrated density from a central interferometer chord and finally the $\mathrm{D}_{\alpha}$ radiation emitted from the outer strike line as ELM monitor. The rate of arriving pellets increases gradually and as a consequence also the associated pellet fuelling. The ELM frequency follows this increase and reaches the highest rate during the phase showing the best pellet performance. Just before the termination of the main heating phase the discharge is subject to additional gas puffing in order to prepare for a soft landing. For the same purpose, some ICRH heating is applied also. It was found in reference discharges that these measures have only a marginal effect on the ELM rate. In the best performance phase, a transient maximum frequency of $32 \mathrm{~Hz}$ is observed, corresponding to a 4.5 times enhancement of the initial rate of $7 \mathrm{~Hz}$. While the pellets, as the only actuator applied, clearly cause this frequency enhancement, neither significant additional fuelling nor confinement degradation takes place. During the steady phase around $11.8 \mathrm{~s}$, a H98 value (H98 quoting the fraction of the plasma energy confinement time predicted by the scaling $\operatorname{ITERH98P}(\mathrm{y}, 2)$ [17]) of about 0.84 was achieved, a value typically found for the low triangularity baseline scenario [11]. 


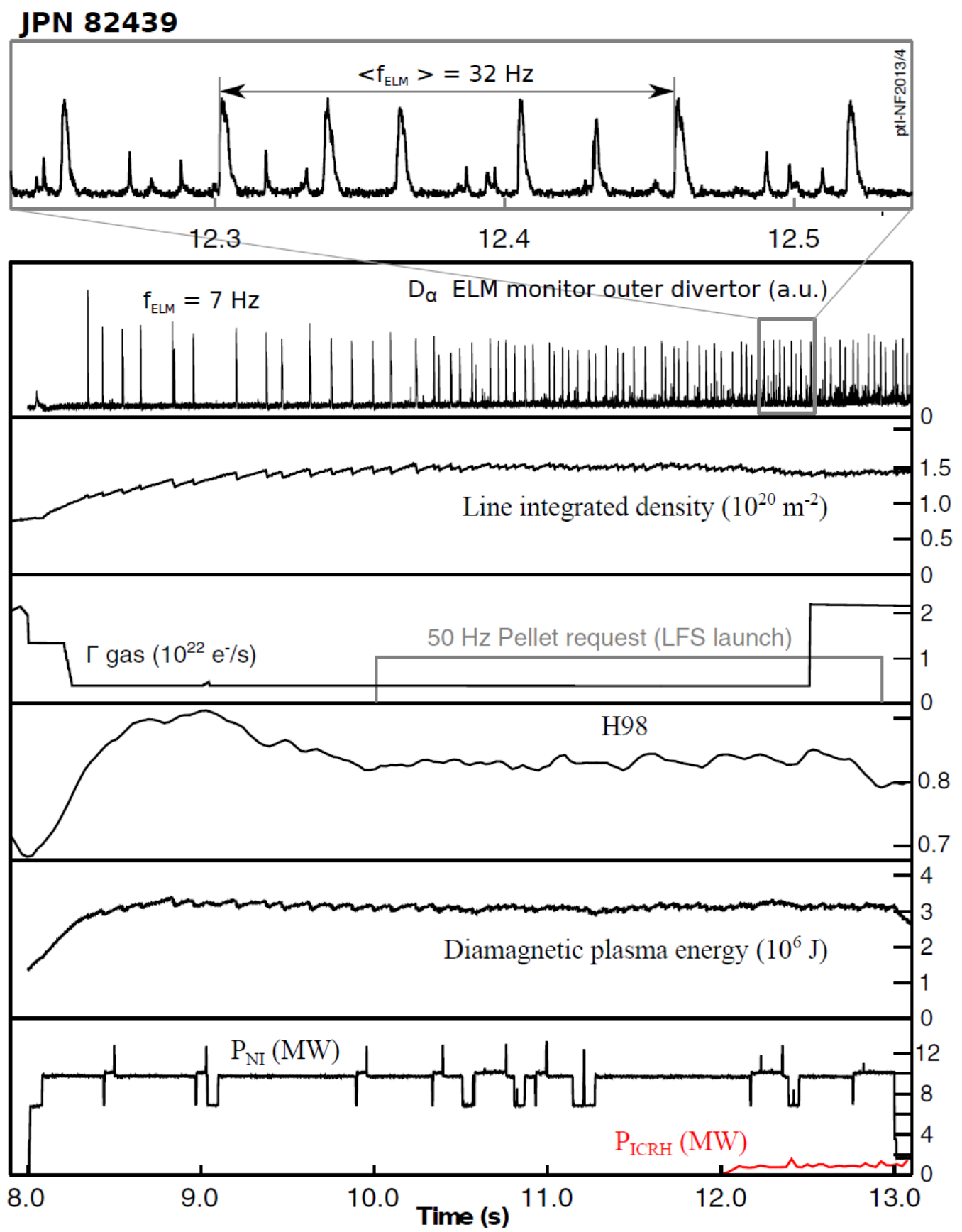

Figure 4: ELM pacing with $50 \mathrm{~Hz}$ small size pellets launched in pacing reference discharge $\left(I_{P}=2.0 \mathrm{MA} B_{t}=2.1 \mathrm{~T}, q_{95}=3.3, \delta^{l}=0.36, \delta^{u}=0.19\right)$. The pellet delivery rate evolves gradually and so does the ELM frequency, finally enhancing the initial rate by 4.5 times.

Because of the pacing pellets showing low operational reliability the more reliable delivered fuelling size pellets were applied for ELM control in the H-mode base line scenario $\left(\mathrm{I}_{\mathrm{P}}=2.5\right.$ $\mathrm{MA}, \mathrm{B}_{\mathrm{t}}=2.65 \mathrm{~T}, \mathrm{q}_{95}=3.3$, high magnetic shaping case with $\delta^{1}=0.36, \delta^{\mathrm{u}}=0.38$ ) where low ELM frequencies must be avoided in order to prevent impurity accumulation. Pellet injection brought the ELM frequency above the pellet rate of $15 \mathrm{~Hz}$, thereby keeping the impurity and radiation level at its low initial level. While for the H-mode base line scenario with lower magnetic plasma shaping $\left(\delta^{1}=0.34\right.$ and $\left.\delta^{\mathrm{u}}=0.19\right)$ it was possible to recover from some early impurity accumulation, the ELM control is required throughout the high performance phase for high magnetic shaping plasmas as shown in figure 5. In a high magnetic shaping reference discharge with the same gas flux of $0.9 \times 10^{22} \mathrm{D} / \mathrm{s}$ but without pellet pacing after a short initial 
phase with about $8 \mathrm{~Hz}$ ELM frequency strong impurity accumulation sets, finally causing a radiative collapse. Using large pellets for pacing causes significant fuelling (estimated about $2.6 \times 10^{22} \mathrm{D} / \mathrm{s}$ ) and hence is expected to results also in considerable convective losses. Nevertheless still confinement characteristics $(\mathrm{H} 98=0.7)$ typical for this scenario and machine configuration [9] could be maintained. Adding a gas puff carrying the same amount of particles instead of pellets, steady conditions can be established at virtually identical plasma energy at an ELM frequency of about $14 \mathrm{~Hz}$.

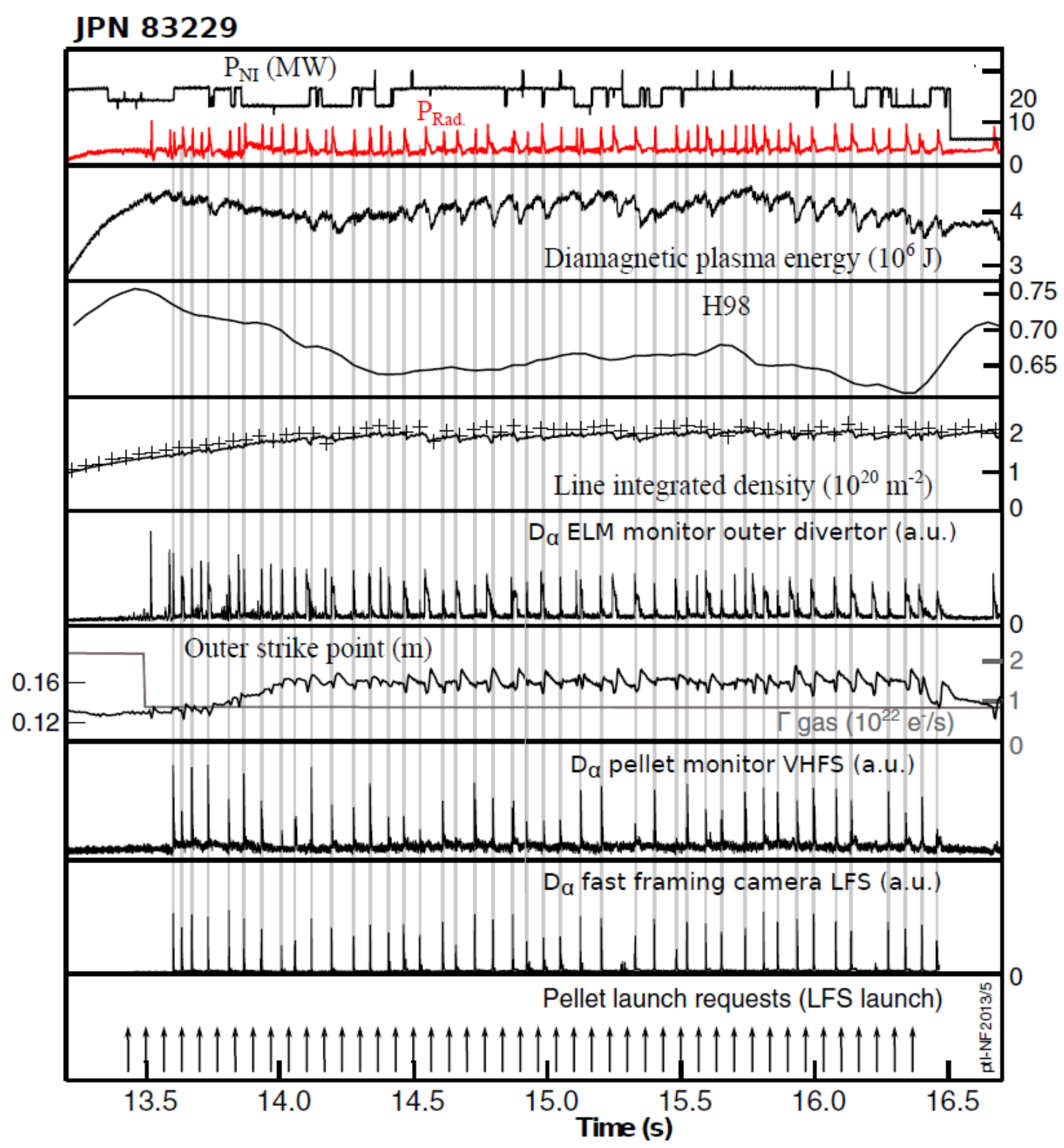

Figure 5: Preventing impurity accumulation by (fuelling size) pellet pacing at $15 \mathrm{~Hz}$ in ITER $H$-mode base line scenario with high magnetic shaping $\left(I_{P}=2.5 M A, B_{t}=2.65 T, q_{95}=3.3, \delta^{l}\right.$ $\left.=0.36, \delta^{u}=0.38\right)$. No confinement reduction is observed.

\section{Triggering investigation}

In the 2.0 MA pacing reference discharges, the pacing pellets are seen to be near the ELM trigger threshold, with the intrinsic mass and speed scatter providing a "free" parameter scan. A statistical analysis of 855 identical requested pellets launched from the LFS at $30-50 \mathrm{~Hz}$ into 14 virtually identical discharges was performed. It turned out that the trigger probability 
(ELMs triggered by a pellet/number of injected pellets) for a fixed plasma configuration depends essentially on three parameters: pellet speed, pellet mass and the time elapsed since the last ELM (spontaneous or triggered). 216 pellets were found to trigger an ELM. The triggering probability versus each of the three single parameters but not discriminating for the other two parameters is displayed in figure 6, showing from left to right: i) time elapsed since the previous ELM (spontaneous or triggered), ii) pellet mass as derived from the fast framing camera and iii) the averaged pellet speed in the final track of the LFS injection path as derived from a time-of-flight measurement. For the chosen configuration with a spontaneous ELM frequency of about $15 \mathrm{~Hz}$, a strong increase of the trigger probability with elapsed time since the previous ELM is, not surprisingly, observed between 10 and $20 \mathrm{~ms}$ during the phase of strongest post-ELM recovery. This is followed by a gradual increase until saturation is almost reached towards the end of the spontaneous ELM cycle. Triggering probability also increases with pellet size and with the measured pellet arrival speed over the observed range of 65 to $110 \mathrm{~m} / \mathrm{s}$.

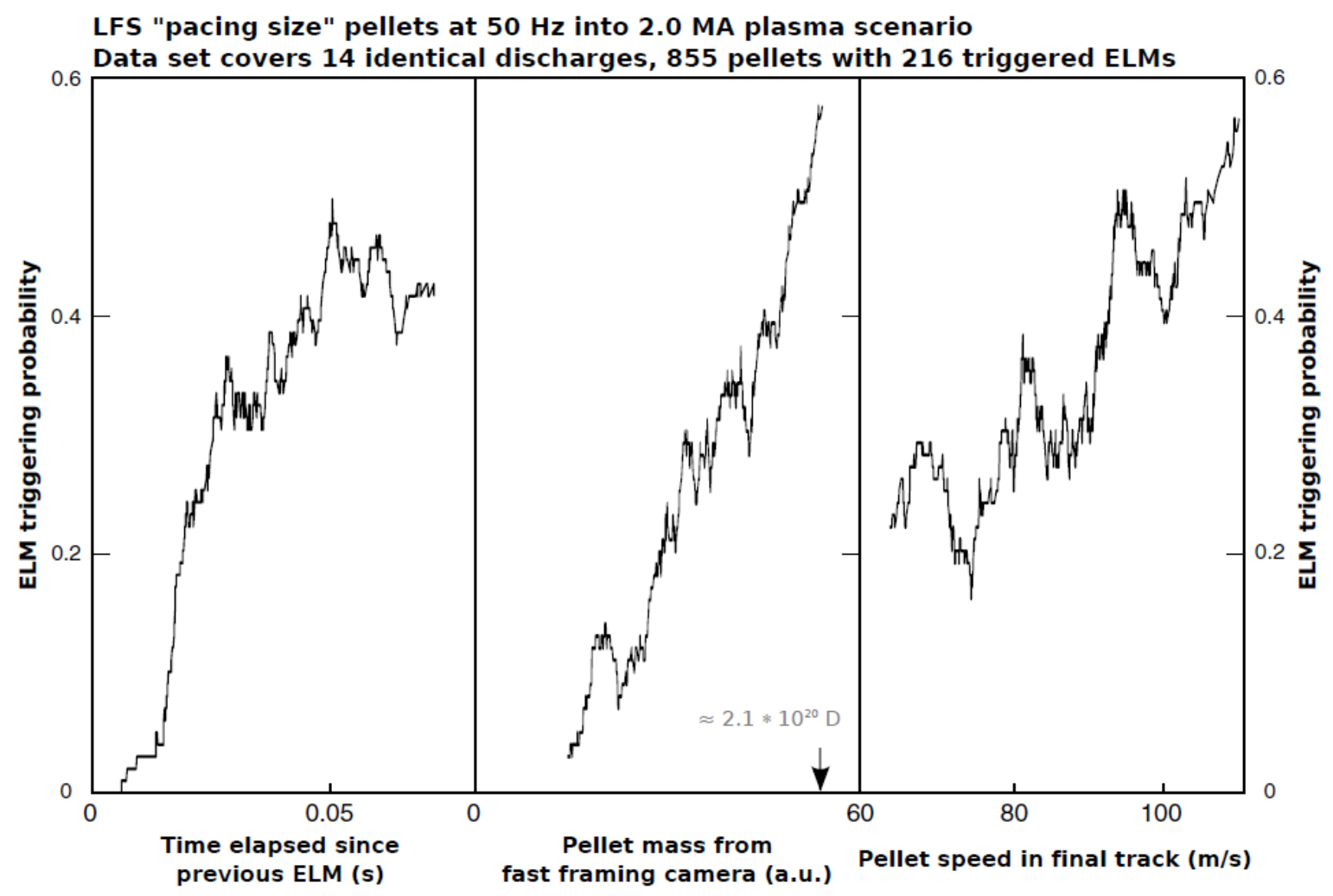

Figure 6: Pellet ELM triggering probability in a 2.0 MA pacing reference discharge with respect to (left to right): i) elapsed time since last ELM, ii) pellet mass and iii) averaged pellet speed in the final LFS track.

Figure 7 is displaying the trigger probability contours as a function of pellet mass and pellet speed. For this analysis, only data points with a time elapsed since the previous ELM of more than $20 \mathrm{~ms}$ were used. Again, the total integrated visible radiation emitted from the particles inside the pellet cloud is used for monitoring the pellet mass. This integrated radiation signal is a monotonic non-linear function of the amount of particles ablated by the pellet and hence can be used to estimate the pellet particle contents. For cases at least $20 \mathrm{~ms}$ after the previous ELM, it becomes evident that the triggering probability approaches unity for pellets approaching the initial design values (arrival with almost full size at a speed of at least 150 
$\mathrm{m} / \mathrm{s}$ ). This confirms that the pellet parameters originally foreseen for the pacing approach are consistent with the request to drive ELMs at a frequency of at least $50 \mathrm{~Hz}$ for this scenario.

\section{LFS "pacing size" pellet data set Time elapsed since previous ELM $>20 \mathrm{~ms}$}

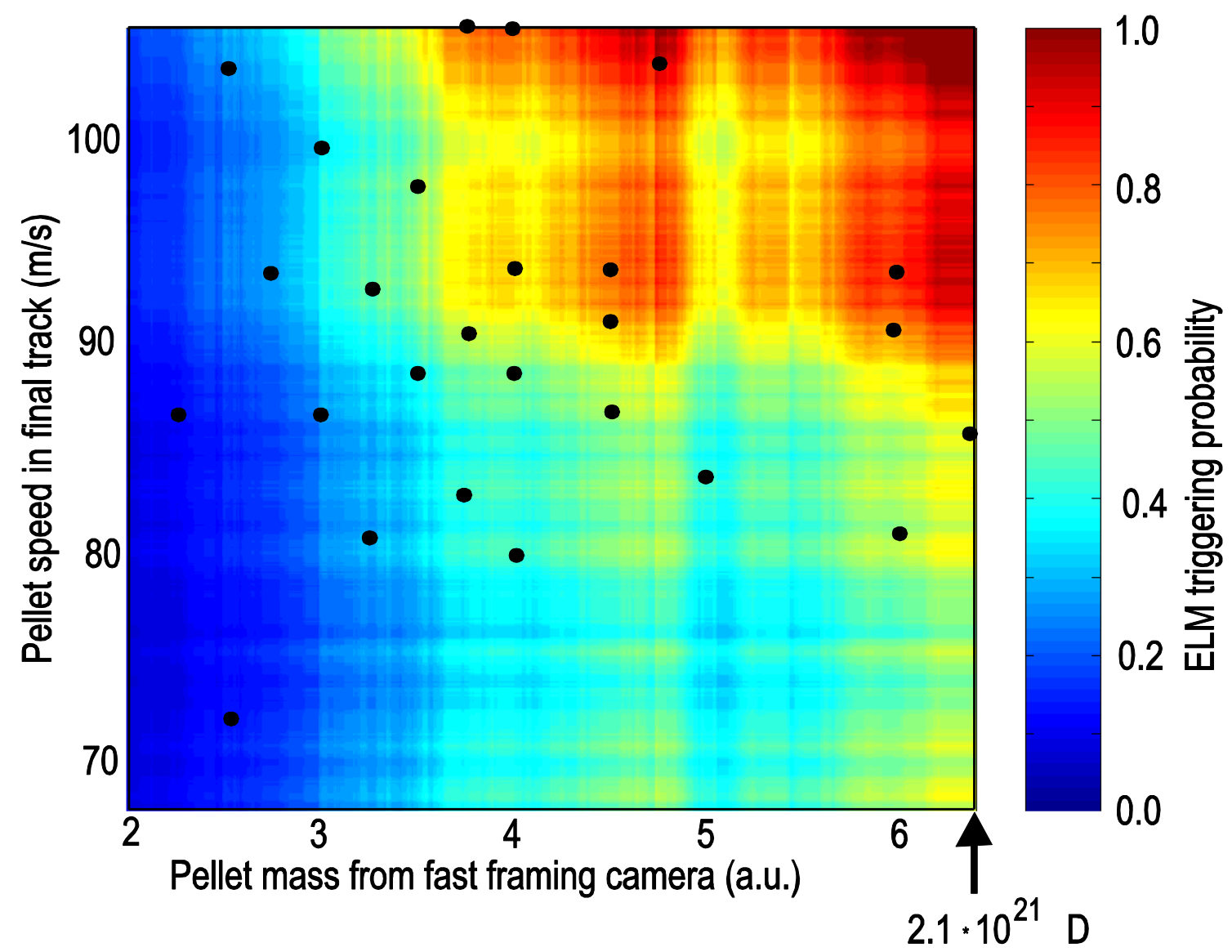

Figure 7: Pellet ELM triggering probability as function of pellet size and speed. Contour lines obtained by fit to data set of 855 pellets, 216 trigger ELMs. Data points (black dots) along probability contour lines selected for analysis of penetration depth.

Along with the probability contour lines at trigger probabilities $0.12,0.25,0.37,0.5,0.63$, 0.75 and 0.87 several pellets (black data points indicated in figure 7) from the data set were chosen for an analysis of the pellet penetration. Penetration was modelled by the pellet ablation and deposition code HPI2 [18] assuming a linear relation between the recorded ablation radiation and the pellet mass and taking the pellet arrival speed to be 0.9 times the average speed in the final track. The analysis was performed the same way as a previous analysis for trigger investigations during experimental campaigns C20 - C27 while JET was operated with a carbon $(C)$ wall $[15,19]$. 


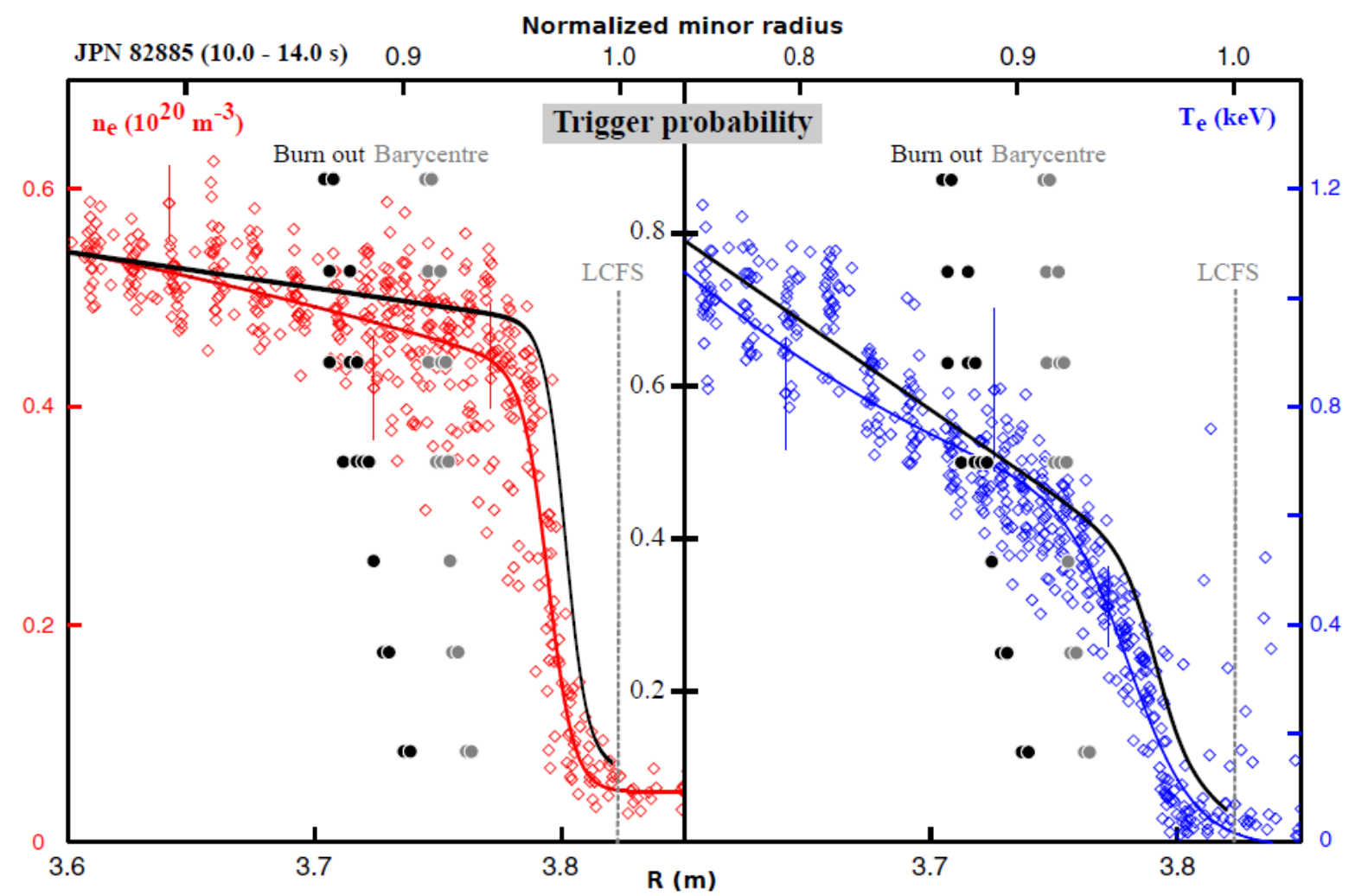

Figure 8: Probability of pellet ELM trigger versus LFS penetration (data shown in figure 7); penetration at full ablation ("Burn out" - black dots) and centre of mass of ablation ("Barycentre" - grey dots). Time averaged (red/blue) and pre-ELM profiles (black) of electron density and temperature.

The result is displayed in figure 8. It shows the triggering probability for the specific mass/speed settings derived from figure 7 as a function of the major plasma radius $\mathrm{R}$ (bottom scale) and the normalized minor plasma outboard radius (top scale) respectively to which the pellet penetrates. The black dots represents "Burn out" the position where the pellet is completely ablated - while the grey dots represent the "Barycentre" - the position of the centre of mass of the ablated particles. For reference the electron density (left) and temperature (right) as measured by the High Resolution Thomson Scattering (HRTS) system are plotted together with modified a tanh-fit to these data (solid coloured line), covering the entire phase of the experiment lasting $4 \mathrm{~s}$ without any correlation to the ELM induced variations. Both solid black lines are obtained from a fit to data points selected for profile measurements immediately before an ELM took place. The difference between the averaged and pre-ELM profiles indicates the strong profile erosion caused by the ELM collapse. For the modelling, both pre-ELM profiles are adopted; hence the penetration depths given here can be regarded as the lower limit of the required penetration in order to achieve the corresponding trigger probability. The exact radial slope of the probability evolution can be distorted slightly by the assumption of a linear relation between the recorded ablation radiation of the fast framing camera and the pellet mass. However, the fact that penetration, by LFS pellet launch, significantly beyond the pedestal top is required to achieve reliable ELM triggering still holds. 


\section{JPN 82885 (10.0 - $14.0 \mathrm{~s})$}

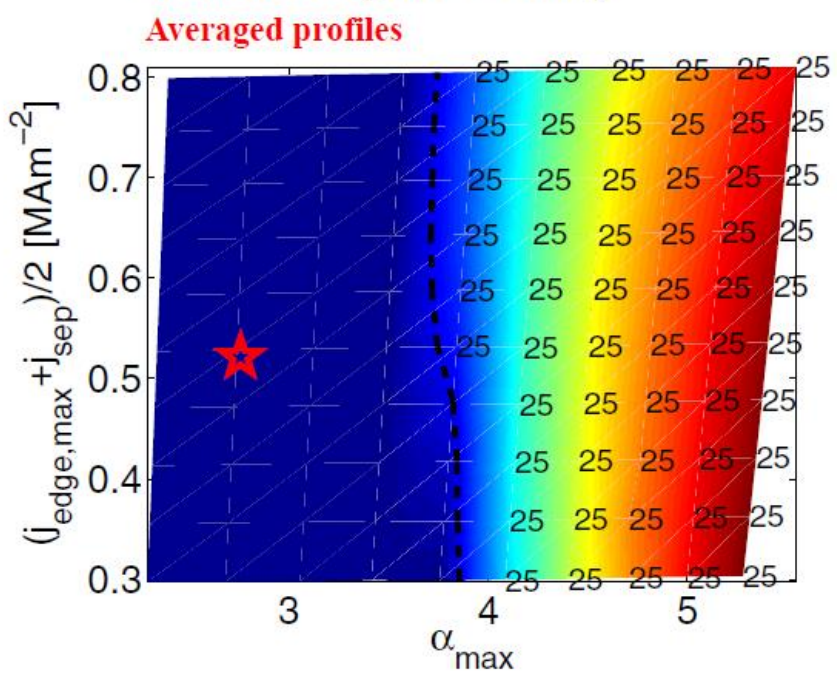

Profiles prior to spontaneous ELM

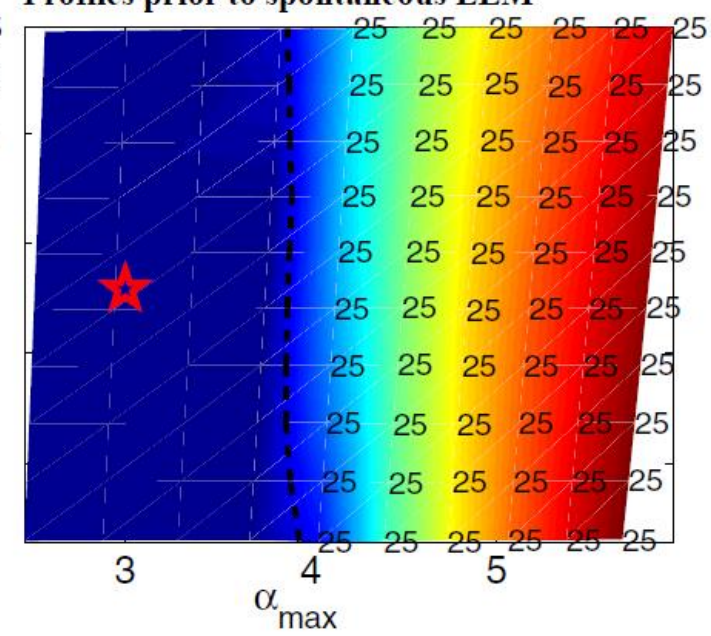

Figure 9: Edge stability diagram of a typical $I_{P}=2.0 \mathrm{MA}$ pacing reference scenario discharge showing spontaneous type-I ELMs. Left: Calculated using averaged profiles (colour lines in figure 8). Right: Profiles just before spontaneous ELM (black lines in figure 8), stars represent the experimental point.

A stability analysis (for more procedure details refer to [20]) was performed taking measurements from the HRTS providing accurate $T_{e}$ and $n_{e}$ profiles in the pedestal region and allowing the derivation of a full electron pressure $\left(\mathrm{p}_{\mathrm{e}}\right)$ profile. These measurements are mapped onto the radial mid plane along flux surfaces provided by the EFIT code and they are then fitted to provide a global analytical fit used in the analysis. As is common, the edge profiles are modelled with the modified hyperbolic tangent function, whose five parameters are used to quantify edge barrier properties. The experimental plasma equilibrium is reconstructed by using the measured $T_{e}$ and $n_{e}$ profiles (assuming $T_{i}=T_{e}$ ) to calculate the bootstrap current self-consistently using the formula from [21]. Using the current and pressure profiles as well as the plasma shape from EFIT reconstruction, the plasma equilibrium is calculated using the fixed boundary equilibrium code HELENA [22]. This equilibrium is then used as a basis for stability analysis with the ELITE code [23] for toroidal mode numbers $n=5-25$. The corresponding experimental points for both analysed cases are represented by the stars in the figures. The boundary for edge stability (dash dotted lines in figure 9) is determined by varying the pressure gradient and the edge current density from the experimental equilibrium. The mode number $n$ of the most unstable mode at the stability boundary gives an indication of the character of the instability (peeling-ballooning $n$ mode number displayed) that is likely to act as an ELM trigger in the plasma. Averaged profiles (as used to produce the left part of figure 9) can be regarded as representing the situation with a relaxed edge profile. It is understood that cases might exist, typically immediately after an ELM crash, with even more relaxed profiles. Globally the edge is in the stable regime, away from the stability boundary. Hence, pellet ELM triggering is attributed to a strong local 3 dimensional perturbation, capable of creating a sufficiently large seed instability evolving into a growing mode despite global parameters still being linearly stable. Pre-ELM profiles for spontaneous events (as used to produce the right part of figure 9) are found to be slightly closer to the stability boundary. However, contrary to experimental observation, the analysis still predicts stability. A similar behaviour was already reported from the stability analysis of some type-I ELMs with a $\mathrm{C}$ wall; possible reasons for this are discussed in the Appendix of [24]. Furthermore, dedicated investigations are in preparation at JET to allow for a closer analysis of this finding. 
Despite the low pellet throughput rate, several discharges were performed with pacing pellets launched from the VHFS. The trigger probability achieved with VHFS launch was compared to the probability obtained with LFS launch for four sets of matched discharges (all basing on the pacing reference scenario). In all cases, pellets had the same requested parameters. Due to the longer guiding tubes associated with VHFS launch, pellets injected via this location are likely to arrive in the plasma with reduced mass and speed as compared to their LFS counterparts. A summary of the four cases is shown in table II. The discharges in the first case includes a small amount of residual gas fuelling while case 2 discharges have been run without any gas fuelling. Eliminating the gas puff is seen to reduce the triggering probability in case of LFS pellet injection. This observation is attributed to an enhanced edge temperature resulting in a reduced pellet penetration. Cases 3 and 4 are discharges like case 1 with kicks (fast vertical motion of the plasma column [25]) added to increase the ELM frequency. Pellets were subsequently injected into these discharges to see the ability of pellets to trigger additional ELMs. A reduction of the probability of pellets triggering ELMs with increasing kick (and ELM) frequency was observed for LFS pellets. This reduction in trigger probability is attributed to the reduced average time elapsed since the previous (mostly kick triggered) ELM at pellet arrival.

As seen from the trigger probability data in table II, VHFS launch shows the same trends as observed for LFS pellets. However, despite the fact that VHFS pellets are likely to be of lower quality than LFS pellets, VHFS pellets seem to have a significantly higher triggering potential than their LFS counterparts. This is also indicated by cases of VHFS pellets triggering ELMs only $10 \mathrm{~ms}$ after the previous ELM, a situation which is very unlikely for LFS launch.

\begin{tabular}{|l|l|l|}
\hline Configuration (Case) & LFS pellet launch & VHFS pellet launch \\
\hline Residual gas (1) & $8 / 18=\mathbf{0 . 4 4}$ & $5 / 5=\mathbf{1 . 0}$ \\
& {$[$ JPN82448] } & {$[$ JPN82449] } \\
\hline No gas (2) & $(20+13) /(63+40)=\mathbf{0 . 3 2}$ & $4 / 5=\mathbf{0 . 8}$ \\
& {$[$ JPN82851+ JPN82852] } & {$[$ JPN82890] } \\
\hline 21 Hz kicks (3) & $6 / 22=\mathbf{0 . 2 7}$ & $3 / 3=\mathbf{1 . 0}$ \\
& {$[$ JPN82882] } & {$[$ JPN83223] } \\
\hline 40 Hz kicks (4) & $7 / 43=\mathbf{0 . 1 6}$ & $3 / 5=\mathbf{0 . 6}$ \\
& {$[$ JPN82848] } & {$[$ JPN83235] } \\
\hline
\end{tabular}

Table II: Comparison of the ELM triggering probability (= ELMs triggered by a pellet/pellets arriving in plasma) for LFS and VHFS launch for different discharges (basing on the pacing reference scenario) but identical pellet request parameters.

These observations fit well to recently reported modelling studies by the integrated core / scrape-off-layer (SOL) / divertor transport code TOPICS-IB [26] showing that small pellets launched from the inboard can trigger an ELM with less pellet mass than similar outboard pellets. The difference arises from the ExB drift of the pellet cloud, producing a wider pressure perturbation around an inboard pellet. Moreover, it cannot be excluded that the acceleration (for VHFS) or deceleration (for LFS) of the pellet plays an important role. This acceleration is due to an asymmetric ablation of the pellet body causing a momentum altering the pellet speed as discussed in [27]. Former fuelling experiments performed at JET showing that inboard launch pellets exhibit a superior core fuelling characteristics over a range of plasma conditions with strong auxiliary heating [28] could be explained based on the underlying processes discussed in [27]. 
With the ILW a significant change of the ELM dynamics is observed for spontaneous type-I ELMs. The ELM duration, for example indicated by the crash of the edge electron temperature and the rise time of the divertor heat load, is much longer for the ILW than in similar cases with a $\mathrm{C}$ wall [9]. The same change is observed for pellet triggered ELMs.

An earlier study showed that any ELM triggered by a pellet under specific plasma conditions shows the same basic features as spontaneous ELMs in the same regime, provided the pellet impact does not change plasma parameters so drastically that even the ELM regime is altered [29]. This behaviour is still true with the ILW in JET where the dynamics of triggered ELMs again is very similar to their spontaneous type-I counterparts. Once more it turns out that the evolution of a triggered ELM follows the same intrinsic dynamics observed for a spontaneous event, indicating that both events are driven by the same instability with the main difference being that a sufficiently strong, externally imposed, pellet perturbation is able to trigger mode growth under conditions where spontaneous growth is unlikely.

JPN 78603 JET-C
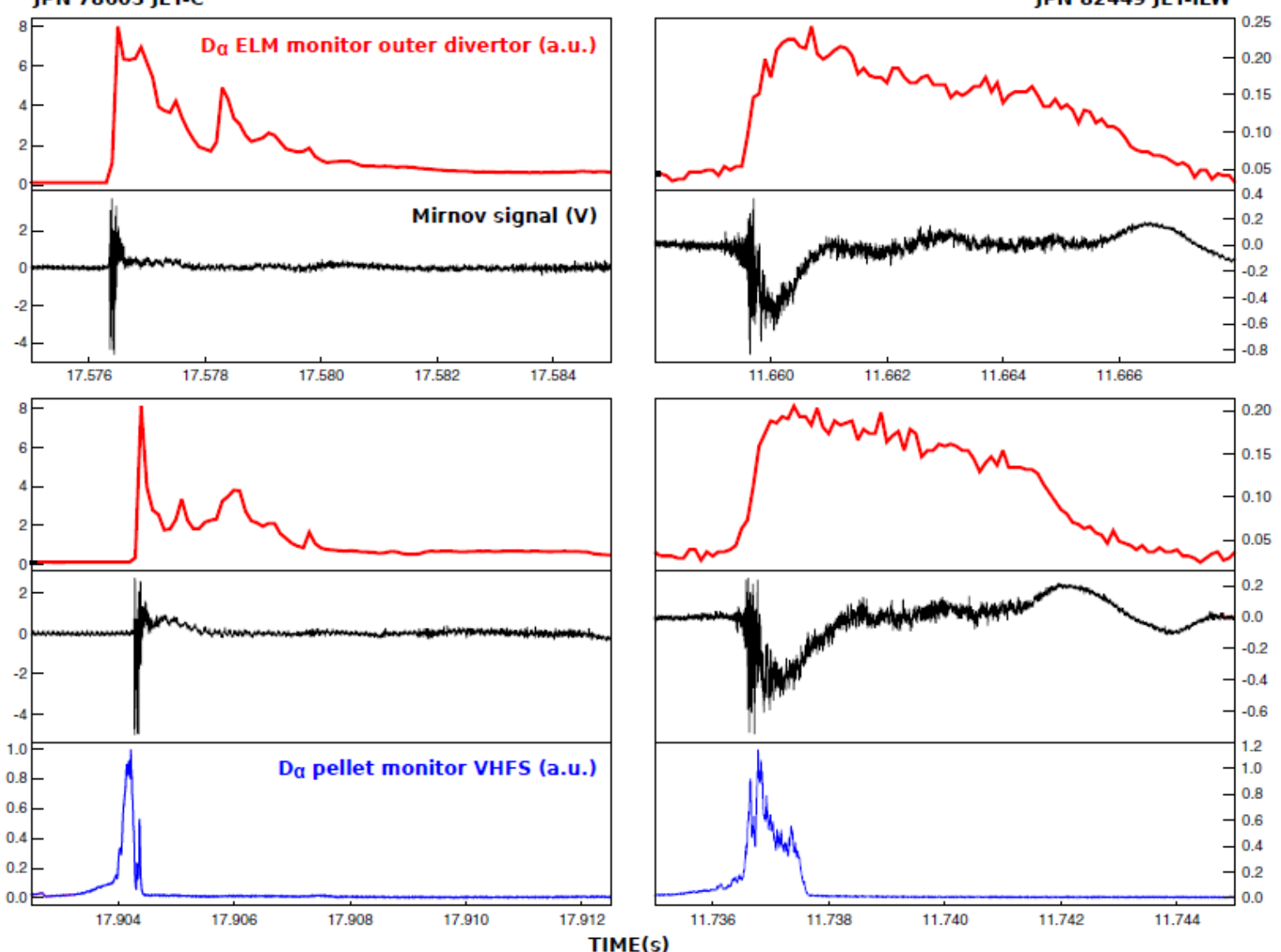

Figure 10: Comparison of spontaneous type-I ELMs (upper frames) and triggered ELMs (lower frames) in a discharge performed during an earlier campaign still operating in a $C$ wall configuration (left frames) to a recent once run with the new ILW (right frames). Signals from top to bottom: i) $D_{\alpha}$ radiation from outer divertor strike point region, ii) MHD activity, iii) pellet ablation radiation (triggered case only).

A comparison of triggered ELMs to their spontaneous counterparts, both for the $\mathrm{C}$ wall (JETC) and the ILW (JET-ILW) is shown in figure 10. Extending a similar analysis performed for JET-C [15] each frame displays (boxes from top to bottom): i) the radiation from the strike line region in the outer divertor indicating the ELM caused power flux evolution; ii) the MHD activity as recorded by a fast pick up coil; iii) the radiation from the pellet ablation region (for the triggered ELM case only). To allow the use of the pellet ablation monitor, which look at 
pellets near the VHFS launch site, VHFS pellets are chosen for this comparison. The two upper frames show the temporal evolution of a single spontaneous ELM, with their corresponding triggered counterparts shown in the lower frames. Frames on the left side represent the $\mathrm{C}$ wall, with the ILW case shown in the frames on the right. Due to the small residual gas puff $\left(0.4 \times 10^{22} \mathrm{e}-/ \mathrm{s}\right)$ required for the ILW case the ELM frequency is $9 \mathrm{~Hz}$, a bit larger than $7 \mathrm{~Hz}$ found in the $\mathrm{C}$ case without puffing. As well, the pedestal temperature and hence the pedestal pressure is significantly lower for the ILW case. For the chosen plasma configuration longer but less intense ELMs appear in JET-ILW compared to JET-C, irrespective of whether the ELM occurs spontaneously or is caused by a pellet. For the ablation radiation, a slight difference is observed, with the radiation lasting longer for the ILW case. This is attributed partially to a longer pellet life time and deeper penetration due to the somewhat reduced edge temperature. Some disintegration of the pellet might also play a role resulting in delayed arrival of debris resulting in the broad second peak.

\section{ELM mitigation?}

Originally, pellet pacing was destined to reduce the thermal stresses on the plasma facing components in the divertor, whilst maintaining better confinement than can be obtained through ELM control by gas puffing [3]. For the assessment of the capabilities of pellet pacing for ELM mitigation, the peak heat flux on the target was studied using infra-red thermography [30]. For this task one first has to know the material limits. One very obvious material limit is the peak heat flux on the target, which can lead to local melting. In earlier studies in machines with a carbon target [30], this quantity was difficult to obtain, due to unknown/changing thermal properties of the target (formation of carbon deposition layers). These shortcomings have been eliminated by going to a tungsten divertor, where no such layers have been observed and by upgrading the existing IR system [31]. For cases with large fuelling pellets the peak heat flux is reduced together with the upstream pedestal pressure (obtained by HRTS). However, this is most likely due to large amounts of particles introduced. With adequate small pacing size pellets, no pedestal pressure reduction is found but no significant reduction of the ELM induced peak heat flux is observed either. Figure 11 compares a paced discharge (JPN82439, left) to a reference discharge without pellets (JPN82447, right). The pellet pacing (setting in at $10.0 \mathrm{~s}$ ) clearly increases the ELM frequency (first boxes) while the relative reduction of the plasma energy per ELM $\Delta \mathrm{W}_{\mathrm{ELM}} / \mathrm{W}$ (last boxes) is reduced by a factor of about 2. A quantity often looked at, when studying ELM mitigation, is the energy $\mathrm{E}_{\mathrm{ELM}}$ deposited by each ELM onto the divertor target plates [31], given here in the second boxes. This quantity is not easily measurable because both the inner and outboard divertor targets have to be observed (which was not possible for the experiments conducted in the ILW due to technical difficulties with the infra-red system). In addition, for ELMs with a large wetted area, not all of the deposited energy can be accounted for, if energy is deposited outside the observed area. A reduction of the $\mathrm{E}_{\mathrm{ELM}}$ on the outer target has been observed in intervals with steady pellet injection. However, the total ELM loss power (ELM deposited energy per time interval) increases when pellet pacing is applied. Hence, the product of ELM deposited energy and ELM frequency is not constant [32].

A more accessible quantity which can be used to assess the ELM induced thermal load is the energy fluency $\varepsilon$, which is calculated by integrating the ELM heat flux over the time duration of an ELM [30]. The result is an energy density deposited by the ELM on the target. For the ELM mitigation experiments, a reduction of the energy fluency by a factor of about 1.5 is observed when increasing the ELM frequency by a factor of 4 . During mitigated phases, however, several ELMs with energy fluencies similar to the unmitigated reference phases are also observed. Hence it seems doubtful if pellet pacing can provide an effective and reliable tool for ELM mitigation in JET. ELM pacing can provide a tool to reduce the deposited energy of single ELMs; however a significant reduction of quantities critical for the survival 
of the plasma-facing components, namely peak heat flux $\mathrm{q}_{\max }$ (third boxes) and energy fluency, has not been observed. Further investigations are needed, and planned, to extend the data bases with respect to this issue. These studies can best be performed by establishing reliable ELM control using small pellets with the ELM frequency increased tenfold.

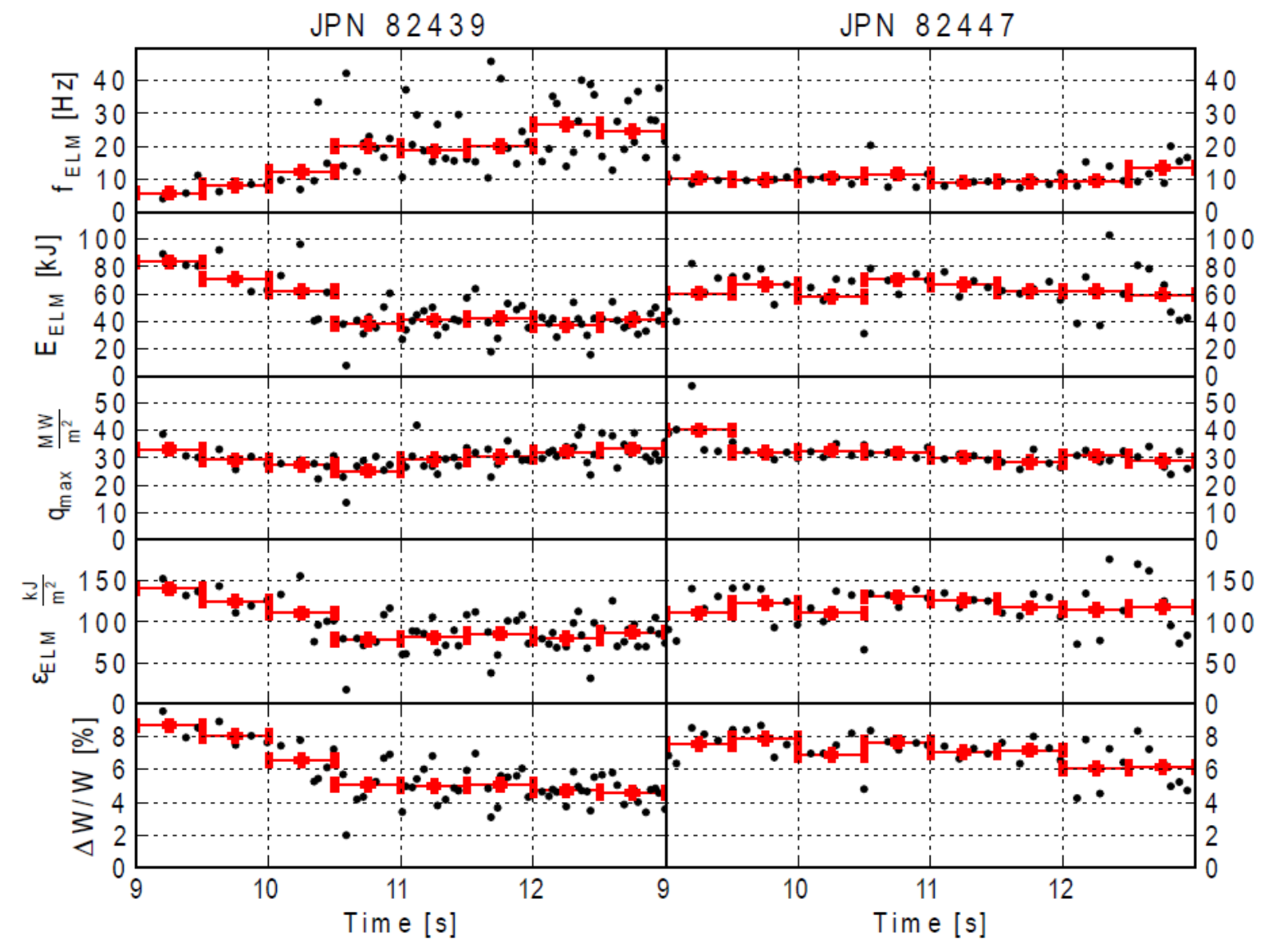

Figure 11: Comparison of paced (left, pellet onset at $10.0 \mathrm{~s}$ ) to reference discharge without pellets (right). Black dots mark individual ELMs; red crosses indicate averaged value of a 0.5 s time interval. (Divertor data correspond to outboard target.)

\section{Discussion and outlook}

Investigations of pellet ELM control and trigger physics have been performed in the new JET ILW environment employing the modernized HFPI. Although the operational capabilities of the pellet launcher are still somewhat limited, good progress was made both in the development of the pellet pacing tool for scenario development and improving the understanding of the physics underlying the ELM triggering process. In particular, the opportunity to compare the first results obtained with the ILW to data from the previous campaigns with the $\mathrm{C}$ wall delivered valuable results.

To achieve reliable ELM sustainment in order to prevent impurity accumulation, only large (fuelling) size pellets at modest repetition rate launched from the LFS could be applied. Despite this, pellet injection was demonstrated to be a useful tool. Since it is expected that sufficiently large and fast pellets can trigger ELMs reliably in discharges with higher energy content and plasma current, pellet ELM control can be employed for the envisaged scenario development towards higher performance.

An increase by a factor up to about 4.5 times the initial ELM frequency was achieved transiently without a noticeable deleterious impact on density or confinement. It was found that small (pacing) size pellets are capable of providing reliable triggering up to the designed 
$50 \mathrm{~Hz}$ pacing rate when reaching the plasma in good shape and at sufficient speed. This seems to indicate that the current operational limitations are due to technical issues and it can be expected that the system will fulfil its prescribed task of a sound persistent enhancement of the ELM rate without introducing significant fuelling once full technical performance is established. Preparations are under way to make the required hardware improvements and improved performance is expected already in the upcoming experiments scheduled for 2013, with further technical improvements planned for 2014.

Taking advantage of the intrinsic speed and mass scatter of the pellets it has been demonstrated that the ELM triggering potential increases with pellet size and speed. Analysis of different cases hints at the resulting variation in pellet penetration being the reason for this. Furthermore, the pellet parameters required to achieve ELM triggering increase when the time elapsed since the previous ELM is decreased. As a consequence it should be expected that there will be a correlation between the achievable ELM pacing rate and the imposed pellet impact.

Significantly deeper penetration, both in absolute distance and normalized to the pedestal width, is required under comparable plasma condition for LFS launch in the JET-ILW compared to JET-C. This might be due to the fact that the degraded pedestal obtained with a $\mathrm{W}$ wall is deeper inside the stability region than in $\mathrm{C}$ wall. Despite the cooler edge favouring pellet penetration in the case of the ILW for fixed pellet parameters, the observed triggering probability is reduced. There is an indication for pellets launched via the VHFS might be superior to those launched via the LFS for ELM triggering. This could be explained by the deeper matter deposit of the inboard pellets due to curvature drift and pre cooling effects. The requirement for a deeper pellet penetration for ELM control in a metal wall environment could potentially have important implications for ITER. Deeper penetration at a maximum possible pellet speed, as restricted by the available guiding systems, can only be achieved by larger pellets. Larger pellets in turn, for a given rate imposed by operational needs, result in a heavier fuelling burden for the de-tritiation system, challenging the capabilities envisaged for this system [33]. Further investigations to substantiate and detail the poloidal asymmetric trigger potential are hence indispensable.

Once again it was found that the dynamics of a triggered ELM are virtually identical to that of its spontaneous counterpart within a specific regime. Changing from a $\mathrm{C}$ wall to the ILW, spontaneous ELMs occurring in a variety of scenarios become less intense but longer lasting and triggered ELMs follow the same pattern. This confirms the previous finding that, once an ELM is triggered by the strong local pellet perturbation - possibly at a time a spontaneous ELM would not occur, it evolves as it's spontaneous counterpart.

Regarding ELM mitigation it seems a significant reduction in plasma energy losses can be achieved with increasing ELM frequency. However, since pellet parameters still varied significantly during the present study this finding has to be reviewed once a better control of pellet speed and size is possible. A much more modest reduction is observed in the energy deposited in the divertor and hardly any reduction is seen in the, more critical, peak heat flux density. Seemingly pellet ELM pacing suffers, as other ELM control methods, like e.g. kick ELM control [25], from the shrinking of the ELM wetted area with increasing ELM frequency [34]. Thus, as is the case when increasing the ELM rate by simple gas puffing, the reduction in energy loss per ELM is not translated into a reduction in the maximum occurring peak heat load. This is in contradiction to recent reports from DIII-D reporting an ELM frequency enhancement by pellet pacing by a factor of 12 with the ELM induced divertor heat flux being reduced by more than a factor of 10 [6]. As ITER depends on proper ELM mitigation for high performance operation [1] this topic clearly needs further investigation including a detailed comparison of different tokamaks and possibly different wall materials. 
Acknowledgments This work was supported by EURATOM and carried out within the framework of the European Fusion Development Agreement. The views and opinions expressed herein do not necessarily reflect those of the European Commission.

\section{References}

[1] A. Loarte et al., " Progress on the Application of ELM Control Schemes to ITER", Submitted to Nuclear Fusion (April 2013).

[2] R. Neu et al., Nucl. Fusion 45 (2005) 209.

[3] P.T. Lang et al., Nucl. Fusion 44 (2004) 665.

[4] R. Dux, A. Janzer, T. Pütterich and ASDEX Upgrade Team, Nucl. Fusion 51 (2011) 053002.

[5] A. Kallenbach et al., Plasma Phys. Control. Fusion 47 (2005) B207-B222

[6] L.R. Baylor et al., $24^{\text {th }}$ IAEA Fusion Energy Conference, San Diego 2012, EX/6-2. http://fec2012.iaea.org/contributionDisplay.py?contribId=755\&sessionId=30\&confId=10 [7] A. Geraud et al., $27^{\text {th }}$ SOFT conference, Liège (Belgium) September 2012, P1.31. http://sciconf.org/soft2012/ip/topic/c/session/p1/paper/31

[8] S. Brezinsek et al., "Fuel Retention Studies with the ITER-like Wall in JET", Submitted to Nuclear Fusion (November 2012).

[9] F. Romanelli et al., "Overview of the JET Results with the ITER-like Wall", Submitted to Nuclear Fusion (November 2012).

[10] S. Brezinsek et al., "Residual carbon content in the initial ITER-Like Wall experiments at JET", Journal of Nuclear Materials (2013), in press; corrected proof online available http://dx.doi.org/10.1016/j.jnucmat.2013.01.122.

[11] R. Neu et al., "First Operation with the JET ITER-Like Wall", 54th APS Meeting Plasma Physics, Providence, Rhode Island (2012), GI2.00002; Phys. Plasma (2013) in press.

[12] G.F. Matthews et al., "Plasma operation with an all metal first-wall: Comparison of an ITER-like wall with a carbon wall in JET", Journal of Nuclear Materials(2013), in press; corrected proof online available http://dx.doi.org/10.1016/j.jnucmat.2013.01.282.

[13] M.N.A. Beurskens et al., 24th IAEA Fusion Energy Conference, San Diego2012, EX/P7-20.

http://fec2012.iaea.org/contributionDisplay.py?contribId=580\&sessionId=30\&confId=10

[14] E. Joffrin et al., 24th IAEA Fusion Energy Conference, San Diego2012, EX/1-1. http://fec2012.iaea.org/contributionDisplay.py? contribId=761\&sessionId=5\&confId=10 [15] P.T. Lang et al., Nucl. Fusion 51, 033010 (2011).

[16] A. Geraud et al., Fusion Eng. and Design 82 (2007) 2183.

[17] ITER Physics Basis Document, Nucl. Fusion 39, 2175 (1999).

[18] F. Köchl et al., $37^{\text {th }}$ EPS Conference on CFPP, Dublin 2010, O4.123.

http://ocs.ciemat.es/EPS2010PAP/pdf/O4.123.pdf

[19] B. Alper et al., $37^{\text {th }}$ EPS Conference on CFPP, Dublin 2010, P2.173.

http://ocs.ciemat.es/EPS2010PAP/pdf/P2.173.pdf

[20] S. Saarelma et al., Plasma Physics \& Controlled Fusion, vol. 53, 085009, 2011.

[21] O. Sauter, C. Angioni C and Y.R. Lin-Liu, Phys. Plasmas 6, 2834 (1999).

[22] G.T.A. Huysmans, J.P. Goedbloed, W.O.K. Kerner, Computational Physics (Proc. Int. Conf. Amsterdam, 1991), World Scientific Publishing, Singapore, 371 (1991).

[23] H.R. Wilson, P.B. Snyder, G.T.A. Huysmans and R.L. Miller, Physics. Plasmas 9, 1277 (2002).

[24] C. Giroud et al., Nucl. Fusion 52 (2012) 063022.

[25] E. de la Luna et al., $24^{\text {th }}$ IAEA Fusion Energy Conference, San Diego 2012, EX/6-1. http://fec2012.iaea.org/contributionDisplay.py?contribId=767\&sessionId=30\& confId=10 [26] N. Hayashi et al., $24^{\text {th }}$ IAEA Fusion Energy Conference, San Diego 2012, TH/5-3. 
[27] H.W. Müller et al., Phys. Rev. Lett. 83, 2199 (1999).

[28] T.T.C. Jones et al., "Results of JET Pellet Fuelling Experiments with Outboard and Inboard Launch", $27^{\text {th }}$ EPS Conference on CFPP, Budapest 2000, OR004.

http://epsppd.epfl.ch/Buda/pdf/or_004.pdf

[29] P.T. Lang et al., Nucl. Fusion 48 (2008) 095007.

[30] T. Eich et al., Journal of Nuclear Materials, Volume 415, Issue 1, p.856-S859

[31] I. Balboa et al., Rev. Sci. Instrum. 83, 10D530 (2012)

[32] T. Eich et al. Plasma Physics \& Controlled Fusion, vol. 49, 573, 2007

[33] R.J. Hawryluk et al., Nucl. Fusion 49 (2009) 065012.

[34] H. Thomsen et al., Nucl. Fusion 51 (2011) 123001. 